\title{
Mental State as a Governor of Human Performance
}

\author{
Commentary
}

Tony Ricci

Dept. of Psychology, Nova Southeastern University, Davie, Florida, United States

Key Words: Perception, Cognition, Anxiety

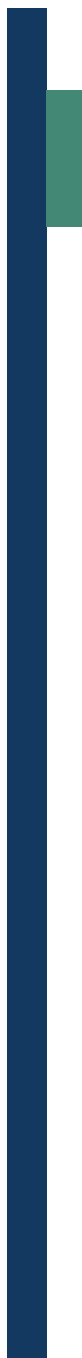

Corresponding author: Tony Ricci, tony@fightshape.net

\section{Commentary}

Open Access

For decades, athletes and coaches have touted the magnitude of the mind and its governance in human performance. Bobby Jones once stated that "golf is a game played on a five-inch course, the distance between your ears." Sport psychologists and mental performance coaches collaborate with athletes in an effort to find, Published: April 28, define, and replicate the optimal mental state when performing. The optimal mental state is often referred to as the "zone", or "flow." This is a state in which an athlete performs to the best of his or her ability"1. While there is some understanding of this mental state, questions remain as to what extent the mind can impact human performance and/or affect physiology. Can one's unwavering belief (perception) in their ability to accomplish a task at hand upregulate physiological qualities that determine such capacities? The breaking of a milestone in human performance, the sub 4:00 minute mile and the subsequent efforts certainly allows us to entertain the aforementioned question.

Roger Bannister of Great Britain became the first human to run a sub 4:00 minute mile at 3:59.4 after nearly 4 years of effort on May $6^{\text {th }} 1954$. This barrier in time was once deemed by many as impossible. His primary competitor in the effort to run the sub 4:00 mile was John Landy of Australia. In 1953 Landy's best times were a 4:02.8 in January and a 4:02.4 in December. In 1954 Landy's mile times undulated again running a 4:02.4 on January $21^{\text {st }}$, then efforts in February resulted in a 4:05.6 and a 4:02.6. Landy again ran in March and April, of that year, with efforts resulting in a 4:05 and a best 4:02.0, respectively. Landy's next attempt would be on June $21^{\text {st }}$, 1954, 46 days after Roger Bannister broke the mark with his 3:59.4. Landy ran a 3:58.0, beating his best time by 4.0 seconds and Bannisters mark by nearly 2 seconds. Those with even a modest understanding of running times could identify that a 4 second reduction in the mile time just 8 weeks after the previous attempt, is more than just a significant improvement; it is highly improbable if one reviews Landy's previous times posted over the forgoing two years. Additionally, while Landy was in peak condition, an accomplished exercise physiologist of modern era would be challenged to explain such advancements in cardiorespiratory, bioenergetics, or biomechanical efficiency from April's 4:02 to the 358.00 on June $21^{\text {st }}$. Moreover, in 1954 the understanding of the aforementioned systems and the capacity to enhance them were quite deficient as compared to our current abilities. Also, it should not be forgotten that Diane Leather became the first female to break the 5:00 mile on May 24th 1954 , just 18 days after Bannister ran his 3:59.4-minute mile. Moreover, the sub 4:00 minute mile was to be achieved numerous times shortly after Bannister and Landy accomplished it. 
Were these run times a logical rate of progression culminating from well-designed training protocols, possibly! Otherwise, might we offer the risky hypothesis that cognition, perception, or simply the optimal mental state, may have favorably altered physiology? That is, did Landy, Leather, and several runners to break the 4-minute mile shortly after Bannisters milestone, do so because they now perceived that the mark could be achieved and they were well suited to do it too?

Conversely, we do have some understanding as to how negative mental states can notably mitigate sport performance. This author has worked with athletes in fight-sports at the highest levels in boxing, kickboxing, and mixed martial arts and experienced the crowning of world titles and several fighters losing their belts. The importance of mental performance became evident after working closely with elite fighters over 10-12 week camps. Throughout the course of training camp, by numerous physiological markers, such as resting heart rates, recovery heart rate, heart rate variability, strength, force production, oxygen consumption, the fighter showed absolute advancements. Moreover, team coaches praised the acquisition of new skill applications and the perfecting of the fighters existing abilities. Nevertheless, within a few minutes into the competition the athlete prematurely fatigued, exhibited poor levels of concentration, poor decision making and sub-standard skill execution. Why and how does this happen? With high probability, the physiology of the central nervous system and periphery was altered in these situations, and certainly not favorably.

As noted, the notion that negative mental states may mitigate performance is widely acknowledged. Unregulated levels of anxiety change physiology and capacities such as cognition, perception, concentration, attention, and motor skills. Anxiety is a negative emotional state characterized by nervousness, worry, and apprehension and associated with activation or arousal of the body. Weinberg \& Gould ${ }^{2}$. While anxiety does not always display as a negative, it manifests in several forms including State and Trait anxiety which may result in disproportionate levels of Cognitive and/or Somatic anxiety. It is the disproportionate levels that often hinder an optimal mental state resulting in declined physical performance.

Neuroscientists, sport psychologists and mental performance coaches agree that unregulated anxiety has both psychological and physiological implications on performance. When anxiety is unregulated it may manifest in hyperarousal of an athlete and to such an extent that he/she finds it hard to concentrate on performance relevant tasks due to constant bombardment on their nervous system. Bali ${ }^{3}$.This state reduces and athlete's ability to examine and judge situations often impairing their decision making. Data also suggests that increased levels of physiological arousal may alter reaction time, movement time and resultant motor functioning in healthy young adults, which comprise the preponderance of the competitive athletic population. Kovacs \& Bories ${ }^{4}$.

While anxiety and hyper-arousal serve as an example as to how performance physiology can be altered via negative cognition and a sub-optimal mental state; then what are the effects on physiology when the athlete is confident or established an optimal mental performance state such as being in the "zone?" There are numerous instances where athletes overcome challenges and odds which are amassed against them. How did John Landy manage to improve his mile time by four seconds after spending well over a year at, and above, 4:02? Does perception in itself change the physiological capacity of the periphery, disinhibit systems normally down-regulated by the brain, or is physiological capacity, to an extent, inhibited until the optimal mental performance state is reached by the athlete?

Much of what we understand about anxiety and negative mental states comes from clinical research and the efforts to treat various disorders, whether they are neurobiological or cognitive in origin. Accordingly, we connect the disorder associated conditions and their effects to derive the potential performance limitations. Martin Seligman proposed the concept of -Positive Psychology- with the premise that the field could go beyond just the clinical treatment of disorders and returning the client to a baseline level of mental health. Seligman proposed from the baseline we can further advance one's mindset and focus on restructuring mental states and cognition in a positive manner so one can flourish, not just return to a baseline mood. Seligman ${ }^{5}$.

While challenges in methodology are plentiful for such research, the future for neuroscientists, sport psychologists and mental performance coaches may warrant investigations into how the optimal mental state, and the zone/flow states, conceivably optimize human performance and physiology. The future training protocols for athletes of all ranks may direct efforts into restructuring neuropathways, cognition and acquiring the athlete's optimal mental state. Of equal importance to strength, speed, and endurance, may be the athlete's perception of these qualities and their certainty in expressing them. 


\section{References}

1. J. Young and M. Pain. The Zone: Evidence of a Universal Phenomenon for Athletes Across Sports. Athletic Insights.1999:1(3).

2. Weinberg, R \& Gould. D. Foundations of Sport \& Exercise Psychology. (6th ed.). Human Kinetics. 2015.

3. Bali, A. Psychological Factors Affecting Sport Performance. International Journal of Physical Education, Sports and Health. 2015:1(6): 92-95.

4. Kovacs, C. \& Bories, T. Effects of Increased Physiological Arousal on Upper Extremity Reaction and Movement Times in Healthy Young Adults. American Journal of Neuroscience. 2010: 1(2): 28-33.

5. Seligman, Martin E. P. Learned Optimism: How to Change Your Mind and Your Life. 2006. New York: Vintage Books. 